\title{
Application of spatial correlation functions in permeability estimation of deformation bands in porous rocks
}

\author{
Anita Torabi, ${ }^{1}$ Haakon Fossen, ${ }^{1}$ and Behzad Alaei ${ }^{2}$ \\ Received 19 October 2007; revised 25 January 2008; accepted 14 February 2008; published 23 August 2008. \\ [1] Millimeter-thick strain localization structures in porous sandstones, known as \\ deformation bands, are reported to perturb the permeability structure of petroleum \\ reservoirs and aquifers. However, the internal permeability structure of deformation bands \\ is difficult to assess by means of classical methods. We have used spatial correlation \\ functions to estimate the porosity and specific surface area in deformed sandstones from \\ high-resolution backscatter images. This work demonstrates the use of a modified version \\ of the Kozeny-Carman relation to calculate permeability based on the information \\ obtained from image processing. We have identified anisotropy with regard to \\ petrophysical properties in deformed sandstones, and demonstrated variations in \\ microstructure and properties across and along the deformation bands. Our results show \\ that properties outside the deformation bands (in the host rock) differ significantly \\ from those inside the band (up to three orders of magnitude). Moreover permeability \\ varies by up to two orders of magnitude along a single band. Furthermore, our porosity \\ and permeability estimates are lower than those obtained from plug measurements. While \\ plug measurements measure the effective permeability across a $2.54 \mathrm{~cm}$ (inch) long \\ sample, the method demonstrated here provides a means to estimate porosity and \\ permeability on the microscale, and to map out the variations in these properties both \\ along and across deformation bands and similar structures.
}

Citation: Torabi, A., H. Fossen, and B. Alaei (2008), Application of spatial correlation functions in permeability estimation of deformation bands in porous rocks, J. Geophys. Res., 113, B08208, doi:10.1029/2007JB005455.

\section{Introduction}

[2] Much attention has recently been devoted to faulted sandstones and their porosity and permeability structure [Ogilvie and Glover, 2001; Bense et al., 2003; Sternlof et al., 2004; Kwon et al., 2005]. Deformation is localized in highly porous sandstones and sediments (i.e., unconsolidated) owing to the formation of deformation bands [Aydin et al., 2006; Fossen et al., 2007], which are millimeter thick zones of deformation. Deformation bands range from compactional through simple shear to dilational, and involve particulate flow, cataclasis and/or dissolution. In most cases, porosity and permeability are altered within deformation bands during formation and growth. In particular, many deformation bands have been found to have considerably lower permeability than their host rocks [Pittman, 1981; Jamison and Stearns, 1982; Antonellini and Aydin, 1994; Knipe et al., 1997; Gibson, 1998; Fisher and Knipe, 2001; Lothe et al., 2002; Shipton et al., 2002], although dilation and permeability increase can occur in some cases [e.g., $D u$ Bernard et al., 2002]. Deformation bands are common constituents of clastic petroleum reservoirs, where they

\footnotetext{
${ }^{1}$ Department of Earth Science and Centre for Integrated Petroleum Research, University of Bergen, Bergen, Norway.

${ }^{2}$ Rocksource ASA, Bergen, Norway.

Copyright 2008 by the American Geophysical Union. 0148-0227/08/2007JB005455\$09.00
}

occur as single structures, as clusters and in fault damage zones [e.g., Hesthammer and Fossen, 2001]. Thus, predicting the role of deformation bands in a petroleum reservoir relies on a sound understanding of their permeability structure.

[3] Permeability is traditionally measured using a minipermeameter in the field or by drilling inch-size cores for laboratory testing. In our experience, miniperm measurements have large uncertainties, and plug measurements are hampered by sampling difficulties, weathering effects and high cost, and require advanced laboratory facilities. Moreover, the resolution of plug measurements is constrained by the plug length (usually $2.54 \mathrm{~cm} / 1$ inch) while deformation structures such as single deformation bands in porous sandstones are only around $1 \mathrm{~mm}$ thick.

[4] As an alternative approach, image processing methods can be used to characterize the microstructure of the rocks and to provide a quantitative means for understanding the dependence of physical properties on the pore structure [Blair et al., 1996]. During the past decades, different approaches have been used to relate microstructural information to physical, mechanical and transport properties of rocks [Ehrlich et al., 1984; Koplik et al., 1984; Wissler, 1987; Doyen, 1988; Blair et al., 1996; Bakke and Øren, 1997; Keehm et al., 2004, 2006; White et al., 2006; Prodanovic et al., 2007]. For instance, Bakke and Øren [1997] presented a process-based model for sandstone that accounts for physical processes such as sedimentation, 
compaction and diagenesis. Their model is suitable only for undeformed sandstone with a limited range of grain sizes. Keehm et al. [2004, 2006] predicted permeability from thin sections. They reconstructed $3 \mathrm{D}$ porous media from $2 \mathrm{D}$ thin section using a stochastic method and the Lattice Boltzmann technique for 3D flow simulation. High resolution X-ray microtomography can be used to characterize the 3D microgeometry of deformation bands if the resolution is sufficient for capturing the complex microgeometry of deformation bands.

[5] We have applied the method of Blair et al. [1996] to the high resolution Backscattered Electron images (BSE) of deformation bands. Moreover, employing high resolution images allows us to capture complex microgeometry in deformation bands. In this study we use spatial correlation functions to characterize the microgeometry of small-scale deformation structures in a statistical way and to estimate their physical properties such as porosity and specific surface area.

[6] Few attempts have been made to apply the spatial correlation functions to obtain the microgeometry of anisotropic porous media. Berge et al. [1997] applied correlation functions for anisotropic porous media on 2D images of tuff. Berryman [1998] has developed the fundamental formulation for stationary anisotropic porous materials, but his concept has not been tested on real anisotropic rock samples.

[7] In the present study, we show how deformation bands induce anisotropy (both across and along the band) to sandstone at the microscale and for the first time apply the concept of Berryman [1998] to a natural anisotropic and heterogeneous medium. Under the Fault Facies research project at the Centre for Integrated Petroleum Research at the University of Bergen, we have investigated many faulted sandstones from different sedimentary basins to explore the effects of deformation bands on the physical properties of sandstone. Understanding the nature of the anisotropy introduced by deformation bands is crucial in estimating three-dimensional properties of deformed sandstones from two-dimensional images. Porosity values have been extracted from low magnification images by applying a one-point correlation function to the binary BSE images of both bands and their undeformed host rock. Specific surface area of the pore-grain interface has been estimated using a two-point correlation function and employing images with different magnifications. The details of three different approaches for calculating specific surface area will be described in the results section. Finally, specific surface area and porosity values were used to estimate the permeability using empirical relations such as the Kozeny-Carman relation. The estimated permeability values are compared to plug measurements.

\section{Theoretical Framework}

[8] Rocks are usually considered as composite materials composed of at least two phases; solids and pores. The properties of composite materials depend on the properties, amount and spatial distribution of each phase. Some properties, such as the elastic modulus and permeability, are functions of pore phase microstructure. The statistical distribution of the relative locations of the microstructural features of interest characterizes their spatial arrangement.

[9] N-point correlation functions are important statistical descriptors that are useful for characterizing the spatial arrangement and heterogeneity of microstructural geometry [e.g., Debye et al., 1957; Corson, 1974; Gokhale, 2004; Cule and Torquato, 1999]:

$$
S_{n}^{(i)}\left(r_{1}, r_{2}, \ldots r_{n}\right)=\left\langle\left(f^{(i)}\left(r_{1}\right) f^{(i)}\left(r_{2}\right) \ldots f^{(i)}\left(r_{n}\right)\right)\right\rangle
$$

[10] Equation (1) describes the volume average over the spatial positions of $r$. N-point correlation functions contain statistical information about the arrangement of the constituents in a composite material by measuring the probability of certain simple geometrical arrangements of the constituents.

[11] The basic information on volume composition and interfacial surface area is contained in the lowest-order correlation functions [Cule and Torquato, 1999]. The onepoint $\left(S_{1}\right)$ and two-point $\left(S_{2}\right)$ pore-pore correlation functions are given by

$$
\begin{gathered}
S_{1}(r)=\langle f(r)\rangle \\
S_{2}(r)=\langle f(r+x) f(r+y)\rangle
\end{gathered}
$$

[12] Two properties of the two-point correlation function can be derived from equations (4) and (5):

$$
\begin{gathered}
S_{2}(r)=S_{1}(r)=\phi \\
r \rightarrow 0 \\
\lim S_{2}(r)=\phi^{2} \\
r \rightarrow \infty
\end{gathered}
$$

[13] $S_{2}(r)$ has a characteristic asymptotic behavior [e.g., Cule and Torquato, 1999]. Grain and pore phases of deformed sandstone occupy different spatial positions. In such a two-phase (grain-pore) system of volume $v$ each phase occupies a subvolume $v_{g}$ (for grains) and subvolume $v_{p}$ (for pores), and a characteristic or indicator function can be defined as follows:

$$
f(\vec{r})=\left\{\begin{array}{ll}
0 & r \in v_{g} \\
1 & r \in v_{p}
\end{array} .\right.
$$

[14] The pore one-point correlation function, $S_{1}$, gives information about the volume fraction of the two phases represented by an image consisting of $\mathrm{M}$ by $\mathrm{N}$ pixels [Garboczi et al., 1999]

$$
\begin{gathered}
S_{1}=\phi=\left\langle f_{(i, j)}\right\rangle=\frac{1}{M x N} \sum_{i j} f_{(i, j)} \\
\mathrm{i}=1,2,3, \ldots \mathrm{M} \text { and } \mathrm{j}=1,2,3, \ldots \mathrm{N}
\end{gathered}
$$



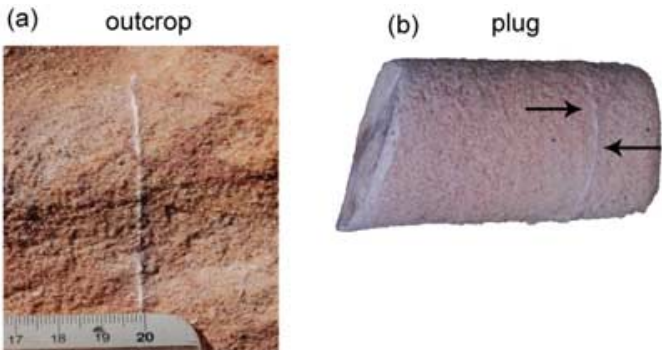

(c) photomicrograph

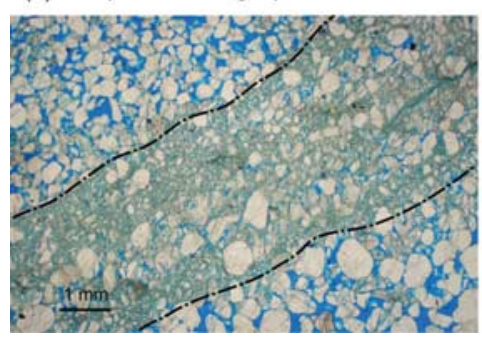

Figure 1. Deformation bands in outcrop (a) plug (b) and under the optical microscope (c).

[15] The pore-pore two-point correlation function, $\mathrm{S}_{2}(\mathrm{x}, \mathrm{y})$, describes the probability that two points with a specified distance apart (a line) are both in pore phase. It is defined by

$$
S_{2}(x, y)=\left\langle f_{(i, j)} f_{(i+x, j+y)}\right\rangle=\frac{1}{M x N} \sum f_{(i, j)} f_{(i+x, j+y)}
$$

[16] We assume that the system is translationally invariant so only the difference between the two pixels is important, not their absolute positions. Furthermore, $\mathrm{S}_{2}(\mathrm{x}, \mathrm{y})$ can be calculated using Fourier transform methods. We have used the following equation [Garboczi et al., 1999] to calculate the two-point correlation function:

$$
S_{2}(x, y)=\sum_{i=1}^{M-x} \sum_{j=1}^{N-y} \frac{f_{(i, j)} f_{(i+x, j+y)}}{(M-x)(N-y)}
$$

[17] The equation shows the two-point correlation function for an $\mathrm{M} \times \mathrm{N}$ matrix (for example a 2D image of faulted sandstone) in a Cartesian coordinate system.

\subsection{Deformation Band Induced Anisotropy}

[18] Characterization of the microstructure of anisotropic porous media has not been explored in much detail and is an important new area within the field of rock physics. The development of more accurate methods that relate anisotropy to rock properties such as permeability is of great importance. Although such applications are more difficult in general, correlation functions may also be applied to anisotropic random media [Berge et al., 1997].

[19] 2D images of thin sections from deformed sandstone have been used in this study. Figure 1 shows typical deformation bands at three different scales: outcrop scale, plug sample scale, and at the microscale represented by a photomicrograph. It is obvious from these pictures that the strain localization represented by the deformation bands adds anisotropy to the sandstone. An anisotropic spatial correlation function of a random porous medium could be used to compute the specific surface area. This can be done by first performing a three-dimensional average over a certain distance $(r)$ and then taking the first derivative with respect to the distance at $r=0$. However, by using 2D images and an understanding of the nature of anisotropy, a planar radial average can be calculated instead [Berryman, 1998]. Therefore we first calculated the two-point correlation function in a Cartesian coordinate system using equation (9). Then we calculated the planar radial average using 2D bilinear interpolation method and the following equation to convert the Cartesian coordinate system to a polar coordinate system.

$$
S_{2}(r)=\frac{1}{2 r+1} \sum_{l=0}^{2 r} S_{2}\left(r, \frac{\pi l}{4 r}\right)
$$

[20] Understanding the nature of anisotropy with regard to petrophysical properties (porosity, permeability) induced by deformation is crucial in estimating three-dimensional properties of deformed sandstones from two-dimensional images. There are both macro- and microscopic aspects of the permeability-related anisotropy caused by deformation bands. Macroscopically, individual deformation bands are only around one millimeter thick and up to a hundred meters long. Fluid flow is reduced across the bands because of the permeability contrast between the bands and their host rock. This aspect has been investigated using plug measurements in the laboratory and mini-permeameter in the field [e.g., Antonellini and Aydin, 1994; Sigda et al., 1999; Shipton et al., 2002]. In addition to these variations across the band, i.e., in the $\mathrm{z}$ direction in Figure 2, microscopic variations within the band may occur in the xy-plane. In order to investigate these microscale variations we have prepared thin sections from two perpendicular sections ( $\mathrm{xz}$ and $\mathrm{yz}$ planes) through a deformation band (Figure 2).

[21] Berryman [1998] assumes that if the random variations of the properties occur only along the $\mathrm{z}$ axis, the surface normal vector is uniformly distributed and lies in the same plane as $r$. Therefore, the specific surface area (i.e.,

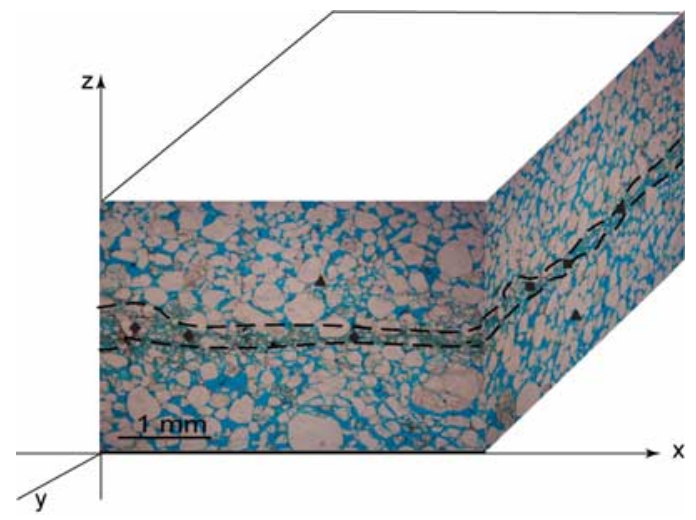

Figure 2. Photomicrographs from two perpendicular thin sections from a deformation band in sample LD-6 showing variation of microstructure along the band in the $\mathrm{xz}$ and $\mathrm{yz}$ planes. The symbols on the Figure correspond to the symbols in Figure 7, which show the approximate location of measured data. 
(a)

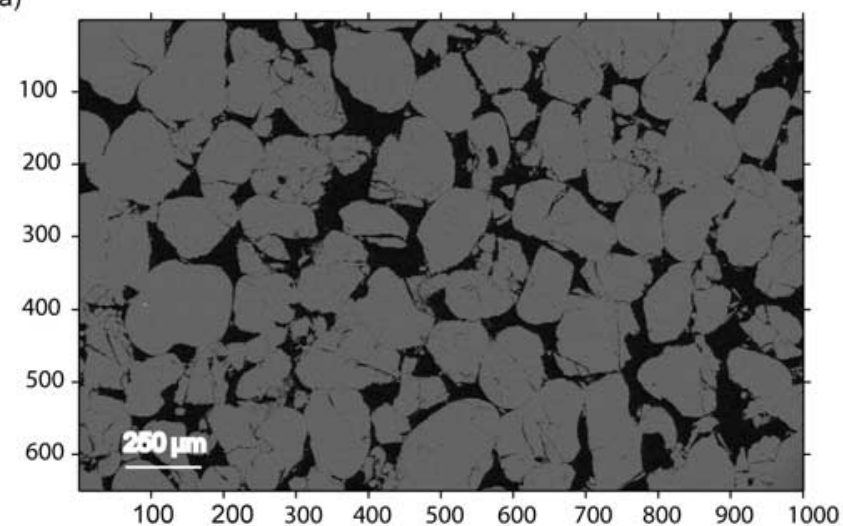

(b)

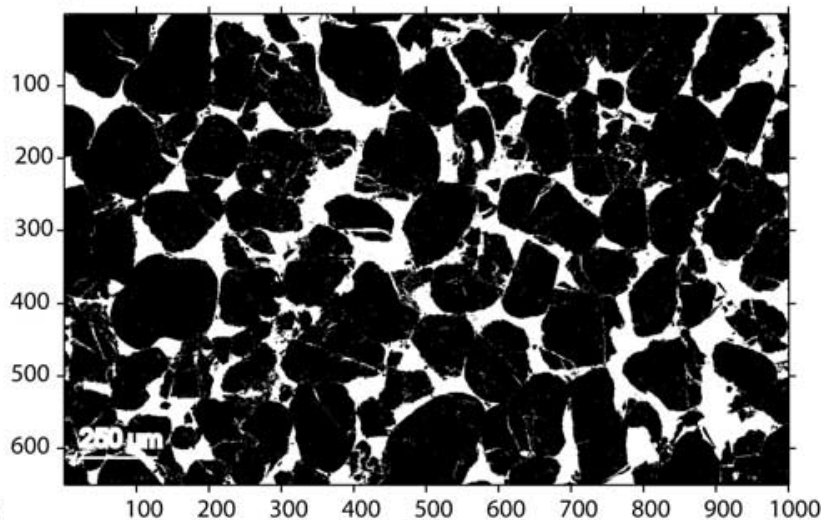

Figure 3. BSE image from the undeformed host rock of sample Wk-01; (a) gray scale image and (b) reversed binary image obtained from the image shown in Figure $3 \mathrm{a}$ with pore space shown in white.

the total area of the pores divided by total volume of the porous media) can be calculated using:

$$
S_{2 x}^{\prime}(0)=-\frac{s}{\pi}
$$

[22] However, this assumption is not always valid since both microstructure and physical properties may vary along deformation bands (Figure 2).

[23] If we consider the deformed sandstone to be anisotropic in three dimensions, then the normal vector does not necessarily lie in the plane of $r$. In this case the specific surface area is given by [Berryman, 1998]:

$$
S_{2}^{\prime}(0)=-\frac{s}{4}
$$

[24] Since we have identified variations in microstructural features such as grain size and pore space distribution along the band (as discussed in more detail in the results section), equation (12) provides a better estimate of the specific surface area from two point correlation functions than equation (11).

\subsection{Image Processing and Spatial Correlation Functions}

[25] The selected BSE images are imported as unsigned 8-bit integer with a gray-scale intensity range from 0 to 255 . The spatial resolution of the image indicates the size of the pixels, with high resolution implying a small pixel size. Both resolution and magnification of the image are important in capturing the microstructural properties of the rocks, as discussed in the results section.

[26] Each image includes a matrix of $\mathrm{M}$ by $\mathrm{N}$ pixels. If there is noise in the data, isolated pixels can be removed by filters such as a median filter. In order to be able to use the indicator function (equation (6)) to characterize the microgeometry of the samples, the color intensity range should be limited to two pixel values representing the two phases of the medium. The intermediate pixels between white and black pixel values in the gray-scale images have proportions of black and white in their areas. When a threshold shade of gray is chosen, all pixels with grays above this threshold are white (grains) and all below are black (pores). Two methods have been used to calculate the threshold value to generate the binary image. The resulting binary image is evaluated by visual inspection and compared to the original BSE image to get the best estimate of the threshold. The two methods are listed below.

[27] (1) MATLAB built-in function (imhist): The function uses the method of Otsu [1979], which chooses the threshold to minimize the intraclass variance of the black and white pixels.

[28] (2) Manual selection: The histogram of intensity range ( 0 to 255 ) versus frequency of each intensity value has been used to select the threshold value manually.

[29] The binary image has pixel value " 0 " in the pore space and " 1 " in the grain space. Since we are interested in void correlation functions, the binary image has been reversed to have " 1 " for voids and " 0 " for grains. Then we define a function $f_{(i, j)}$ that is zero for grains and one for pores, and $(i, j)$ indicates the position of pixels in the image. Figures $3 \mathrm{a}$ and $3 \mathrm{~b}$ show an original gray-scale image and its reversed binary image (pores white and grains black). The one-point correlation function has been calculated for the reverse binary image using equation (7).

[30] In this work, equations (7), (9) and (10) have been used in a function that is written in MATLAB to calculate the one- and two-point correlation functions for the reversed binary image respectively. Finally equation (12) has also been used to estimate the specific surface area from two-point correlation functions. Figure 4 shows the anisotropic twopoint correlation function variations versus $r$. The two properties of the two-point correlation function (equations (4) and (5)) and its asymptotic behavior are also shown in Figure 4. The slope of the planar radial average two-point correlation function at $r=0$ (straight line in Figure 4) provides an estimate of specific surface area (equation (12)).

\subsection{Permeability and Kozeny-Carman Relation}

[31] In order to estimate permeability we use a modified version of the Kozeny-Carman relation developed by Paterson [1983] and Walsh and Brace [1984]. Here, pores 


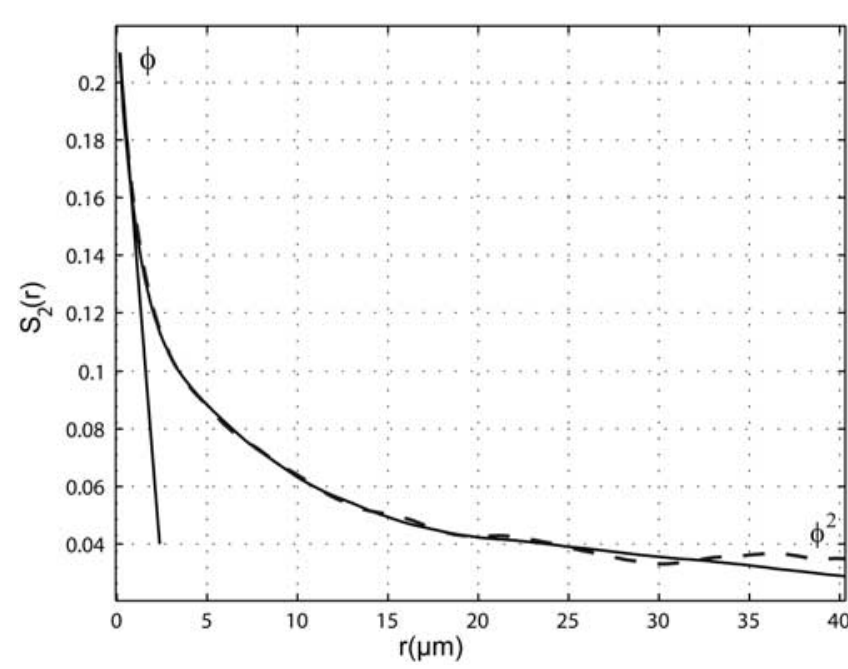

Figure 4. Solid line illustrates calculated planar radial average two-point correlation function versus distance. The straight line shows the slope of the two-point correlation function at the origin $(r=0)$. For comparison, calculated two-point correlation function versus distance (dashed line) at $x=1$ and $y=1$ (equation (9)) before taking the average is shown. Note the properties that can be obtained from twopoint correlation function. The maximum value at the origin is equal to porosity and the asymptotic value corresponds to $\varphi^{2}$.

in porous media are considered to be tabular. We then have that

$$
k=\phi^{2} / c F s^{2}
$$

where $\phi$ is the porosity, $c$ is a constant related to pore geometry that is equal to 2 for materials possessing pores of circular cross-section, and $F$ is the formation factor and has an exponential relationship with porosity [Archie, 1942];

$$
F=\phi^{-m}
$$

[32] Furthermore, $s$ is the specific surface area of the tubes. Archie [1942] defined an empirical relationship between electrical properties and formation factor for rocks. Later Wyllie [1953] developed the relationship between formation factor and other properties of rocks, such as porosity. In this relation the exponent $\mathrm{m}$ is a cementation factor and it varies between 1.5 for poorly consolidated sandstones and 2 for well consolidated sandstones [Brace, 1977; Sen et al., 1981; Wong et al., 1984; Blair et al., 1996]. Our host rock samples are poorly consolidated with a very low degree of cementation. Therefore we have chosen 1.6 for the exponent $\mathrm{m}$. As results of cataclasis, pressure dissolution, and low degree of cementation, the deformed samples have become moderately consolidated. Hence, we have chosen 1.8 for the deformation bands. The computed porosities and specific surface areas from spatial correlation functions are used to calculate permeability using equation (13).

\section{Results}

\subsection{Image-Based Analyses}

[33] The size range resolvable from backscatter images depends on the magnification and resolution of the image. We have used BSE images $(1024 \times 768)$ with variety of magnifications, i.e., from $155 \times$ up to $2580 \times$. It is important to consider the effect of magnification on the calculated one- and two-point correlation functions. On the basis of previous studies [e.g., Blair et al., 1996], low magnification images (images which include 10-100 grains) seem to be appropriate for calculating one-point correlation functions (porosity), since they cover a larger area of the sample than high magnification images and therefore are more representative. It has also been verified that laboratory measured porosity values are in agreement with the estimated values from low magnification images. On the other hand, high magnification images are more suitable for obtaining a twopoint correlation function and hence specific surface area of the pore-grain interface [e.g., Blair et al., 1996].

[34] Having accepted the concept of Blair et al. [1996], we use low magnification images that cover the whole area of interest for porosity estimates from both inside and outside the band (in the host rock). To calculate specific surface area in the host rock by means of the two-point correlation function, we use a high magnification image. Cataclastic deformation bands have a wide range of grain size and pore geometry. Therefore it is a challenge to pick the appropriate magnification to assess the accurate specific surface area of the pores in these bands. This challenge will be addressed later in the paper.

\begin{tabular}{|c|c|c|c|c|c|c|}
\hline Sample & Grain Size & Mineralogy & Type of Band & Formation & Location & UTM Coordinates \\
\hline UT & fine & quartz arenite & $\begin{array}{l}\text { cataclastic band with } \\
\text { quartz dissolution }\end{array}$ & Entrada Sst. & $\begin{array}{c}\text { San Rafael Desert, } \\
\text { Utah, USA }\end{array}$ & 0525998-4271679 \\
\hline $\mathrm{CNM}$ & fine & quartz arenite & $\begin{array}{l}\text { cataclastic band with } \\
\text { quartz dissolution }\end{array}$ & Navajo Sst. & $\begin{array}{l}\text { Colorado Nat'1 } \\
\text { Mon., USA }\end{array}$ & $4332902-695343$ \\
\hline $\mathrm{V}$ & fine to medium & quartz arenite & $\begin{array}{l}\text { cataclastic band with } \\
\text { some quartz } \\
\text { dissolution }\end{array}$ & Moab Mbr. & $\begin{array}{l}\text { Hidden Canyon } \\
\text { Utah, USA }\end{array}$ & $4285800-605825$ \\
\hline Wk-01 & medium & quartz arenite & cataclastic band & Nubian Sst. & Sinai, Egypt & 0523668-3219513 \\
\hline Wk-08 & medium & quartz arenite & cataclastic band & Nubian Sst. & Sinai, Egypt & $0523668-3219513$ \\
\hline I & medium & quartz arenite & $\begin{array}{l}\text { cataclastic band with } \\
\text { some iron oxide } \\
\text { cement }\end{array}$ & Nubian Sst. & Sinai, Egypt & $\ldots$ \\
\hline LD-6A, B & medium & quartz arenite & $\begin{array}{c}\text { cataclastic bands with } \\
\text { some iron oxide } \\
\text { cement }\end{array}$ & Nubian Sst. & Sinai, Egypt & $\cdots$ \\
\hline
\end{tabular}

Table 1. Description of the Samples of the Thin Sections Used in this Study 
(a)

(b)

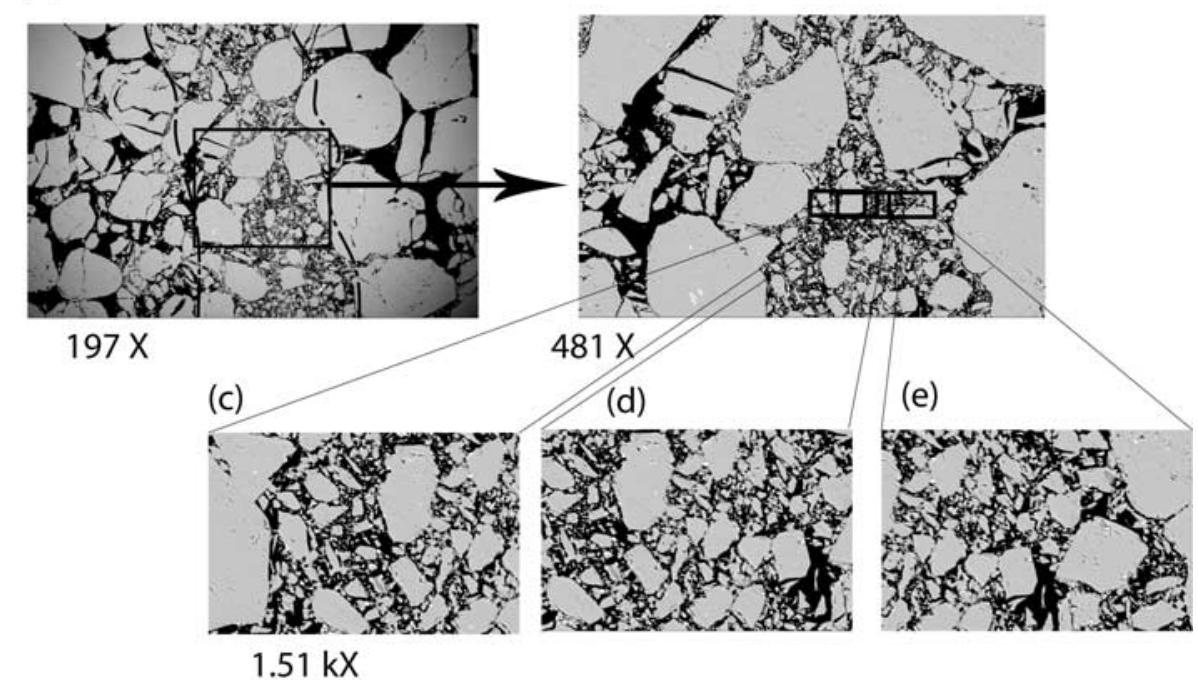

Figure 5. (a) BSE image from deformed sandstone with a central deformation band. (b) Low magnification BSE image from the band that covers the entire width of the band and has been used for porosity estimation of the band. (c), (d), (e) High-magnification BSE images taken across the band that cover the entire width of the band and have been used for calculation of two point correlation functions and hence average of the specific surface area. Although the high magnification images overlap, the nonoverlapping parts have been selected for calculation of two-point correlation functions.

[35] Table 1 shows the information related to the samples that have been used in this study. Figure 5 shows five BSE images from sample Wk-01 from Sinai, Egypt at different magnifications as examples. Figure 5a illustrates an overview of the whole thin section from sample Wk-01, which has a deformation band in the middle. By increasing the magnification (Figures $5 \mathrm{c}, 5 \mathrm{~d}$, and $5 \mathrm{e}$, which have been used for calculation of specific surface area) only a small part of a deformation band is perceptible and hence the calculated specific surface area is not representative. Our research shows that in order to capture the complex microgeometry of deformation bands we need several highresolution and magnification BSE images across the band. In order to verify this idea and examine the two-point void correlation function sensitivity to the magnification of the images, a database from our measurements for deformation bands, using images with different magnifications from deformed sandstone is provided (Table 2 and Figure 6). We applied three different approaches.

[36] (1) First we calculated the specific surface area of the pores from an image with a low magnification that covers the entire thickness of the band. We have also used this image for porosity estimation since it includes the wide variety of the grain and pore size in the deformation bands (squares in Figure 6 show the calculated permeability using this approach).

[37] (2) In a second approach, we followed the concept of Blair et al. [1996] and used one high magnification image from a small part of the band to calculate specific surface area of the pores. Triangles (samples from the deformation bands) and stars (samples from the host rocks) in Figure 6 show the calculated permeability using this approach.

[38] (3) In a third approach, several high magnification images were taken across the band to cover the entire thickness of the band. Then the average specific area for the entire band was calculated using specific surface areas obtained for the different images (diamonds in Figure 6 show the calculated permeability using this method).

[39] The permeability values calculated by using the first approach are always higher than those from the next two approaches. We can classify our results into two different groups.

[40] (1) In samples "Wk-01", "Wk-08" and "I" from Sinai, the permeability values obtained from averaging the

Table 2. Calculated Porosity, Specific Surface Area, and Permeability Using the Three Different Approaches Discussed in the Text

\begin{tabular}{lcccc}
\hline & & & & Calculated \\
\multicolumn{1}{c}{ Sample } & Approach & $\begin{array}{c}\text { Specific Surface } \\
\text { Area, } \mu^{-1}\end{array}$ & $\begin{array}{c}\text { Porosity, } \\
\%\end{array}$ & $\begin{array}{c}\text { permeability, } \\
\text { mD }\end{array}$ \\
\hline UT-host & 2 & 0.039 & 31 & 5049 \\
Wk-01-host & 2 & 0.037 & 21 & 1300 \\
Wk-08-host & 2 & 0.022 & 23 & 5731 \\
V-host & 2 & 0.048 & 29 & 1632 \\
CNM-host & 2 & 0.054 & 24 & 1006 \\
I-host & 2 & 0.059 & 24 & 833 \\
UT-band & 1 & 0.084 & 9 & 11 \\
UT-band & 2 & 0.091 & 9 & 9 \\
UT-band & 3 & 0.124 & 9 & 4 \\
Wk-01-band & 1 & 0.13 & 14 & 16 \\
Wk-01-band & 2 & 0.405 & 14 & 1.7 \\
Wk-01-band & 3 & 0.349 & 14 & 3 \\
Wk-08-band & 1 & 0.178 & 17 & 17 \\
Wk-08-band & 2 & 0.31 & 17 & 5 \\
Wk-08-band & 3 & 0.386 & 17 & 6 \\
V-band & 1 & 0.054 & 7 & 20 \\
V-band & 2 & 0.07 & 7 & 13 \\
V-band & 3 & 0.1 & 7 & 2 \\
CNM-band & 1 & 0.15 & 11 & 6.9 \\
CNM-band & 2 & 0.17 & 11 & 5 \\
CNM-band & 3 & 0.217 & 11 & 2 \\
I-band & 1 & 0.16 & 15 & 1.7 \\
I-band & 2 & 0.6 & 15 & 15 \\
I-band & 3 & 0.585 & & 2.2 \\
\hline
\end{tabular}




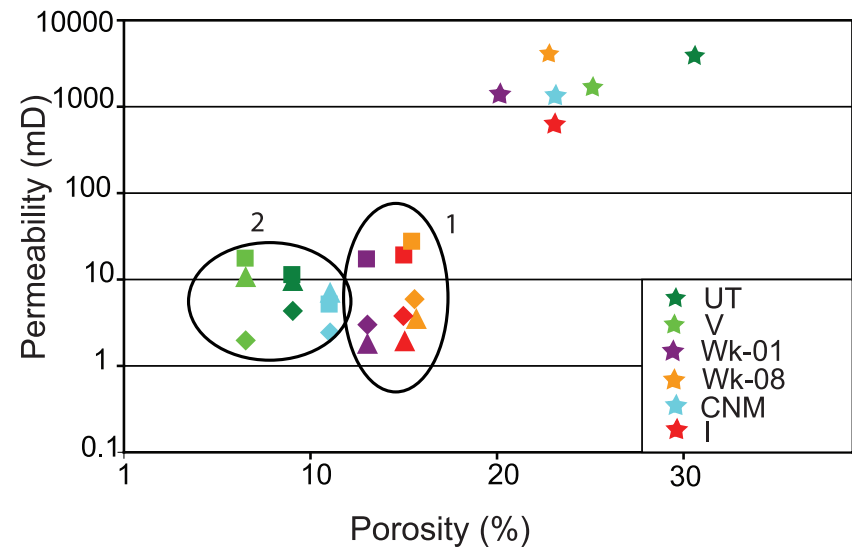

- Deformation band; approach 1 was applied.

\ Deformation band; approach 2 was applied.

- Deformation band; approach 3 was applied.

- Host rock; approach 2 was applied.

Figure 6. Permeability versus porosity as estimated by using the image processing method described in the text. For permeability estimation, specific surface area was calculated through three different approaches: Approach 1 (squares): A low magnification image that covers the entire thickness of the band has been used to calculate specific surface area. All the porosity measurements were calculated by applying this approach. Approach 2 (triangles): An image with higher magnification was used to calculate specific surface area. This image covers a part of the band. Approach 3 (diamonds): An average specific surface area has been calculated using several high magnification images. Note the contrast in porosity and permeability within the bands and host rocks (stars) and also the classification of data for deformation bands into two groups. Group 1 includes the samples from Sinai, where cataclasis is the main deformation mechanism and group 2 includes samples from Utah, where both cataclasis and some pressure dissolution are involved. For more detail see Figure 5 and Table 2.

specific surface area (approach 3) are lower than the results from the first approach but higher than the values from the second approach.

[41] (2) In sample "UT", "CNM" and "V" from Utah, the permeability values from approach 3 are lower than values obtained from approaches 1 and 2 .

[42] Figure 6 also illustrates that both porosity and permeability have been reduced within the bands. The highest porosity reduction was found for sample UT, where porosity is reduced from $\sim 30 \%$ outside the band to $\sim 10 \%$ within the band and permeability is decreased by up to three orders of magnitude. This variation comes from the first source of anisotropy (macroscopic aspect) associated with the deformation band, as discussed in section 2.1. To quantify the microscopic aspect of anisotropy along deformation bands, we have estimated porosity and permeability along a single deformation band (Sample LD-6 from Sinai, Egypt) in two perpendicular thin sections (Figures 2 and 7). In this sample, we have applied our third approach for the deformation band and second approach for outside the band to calculate permeability. Porosities in the xz-plane (diamonds in Figure 7) are smaller than porosities in the yzplane (about 15\% differences). While, permeability values in the xz-plane are up to two orders of magnitude lower than those in the yz-plane.

\subsection{Laboratory Measurements and Their Comparison With the Calculated Data}

[43] In the present study, core plugs of $2.54 \mathrm{~cm}$ ( 1 inch) in diameter and length have been used for porosity and permeability laboratory measurements. The core-plugs were taken perpendicular to the deformation bands. A gas-transfer technique has been used for both porosity and permeability measurements, and a Helium porosimeter was used for the porosity measurements. The instrument works based on the principle of Boyle's law ( $\mathrm{PV}=$ constant, where $\mathrm{P}$ is the pressure and $\mathrm{V}$ the volume). Gas permeability was determined by flowing gas through the samples. At steadystate conditions, the gas flow rate, the pressure drop over the plug sample and the upstream pressure were recorded. The confining pressure applied during the measurement was 20 bar. The gas permeability $\mathrm{K}_{\mathrm{g}}$ was then calculated using Darcy's law [Darcy, 1856]. The Klinkenberg corrected permeability value, $\mathrm{K}_{1}$, was determined empirically, based on the measured $\mathrm{K}_{\mathrm{g}}$ and the corresponding average pore pressure, $\mathrm{P}_{\mathrm{m}}$ [Klinkenberg, 1941].

[44] We compared our estimated porosities and permeabilities to laboratory measured data obtained from plugs of the same samples. Most of our calculated permeability for deformation bands is slightly lower than the laboratory data, regardless of the choice of image processing approach (Figure 8). The maximum difference between the estimated and lab-measured porosity is $9 \%$ that is for the sample with the lowest porosity (Figure 8). The calculated permeabilities from the third approach are up to two orders of magnitude less than the laboratory measurements.

\section{Discussion and Conclusions}

[45] Since deformation bands are small-scale structures with about 1 millimeter thickness it is a challenge to obtain their internal properties precisely. Both field and laboratory

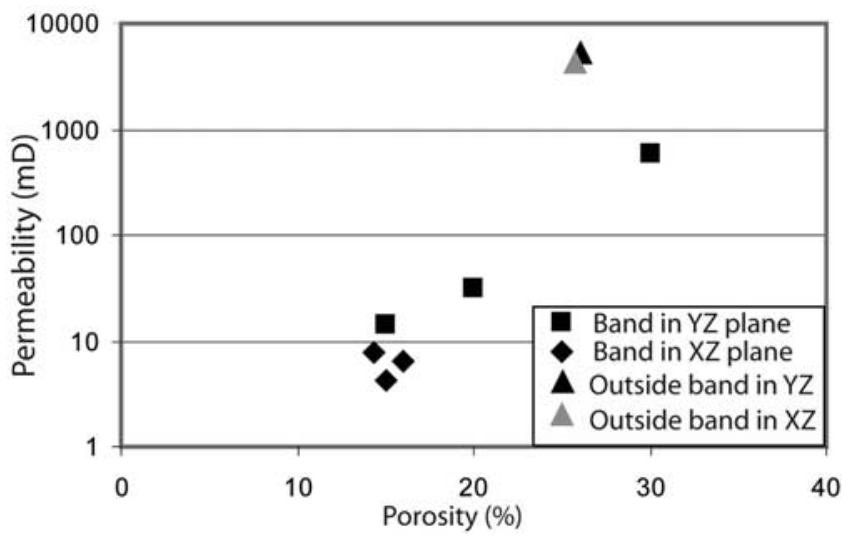

Figure 7. Porosity and permeability obtained from two perpendicular thin sections (xz and yz, see Figure 2) from sample LD-6 (Sinai, Egypt). Note the variations along the band. 
(a)

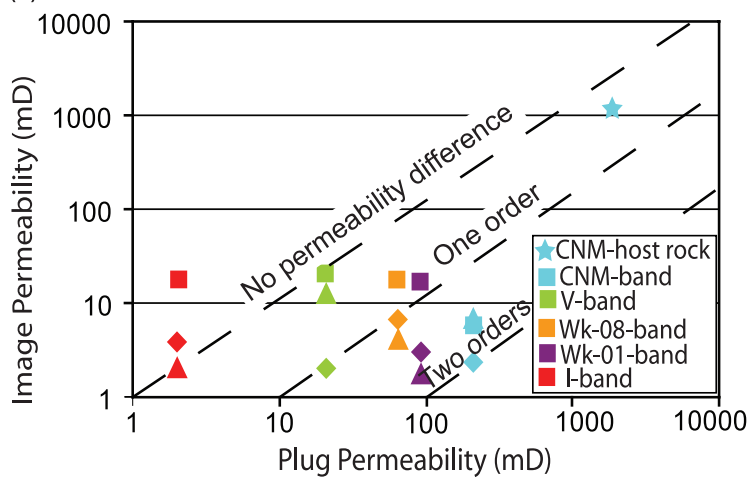

(b)

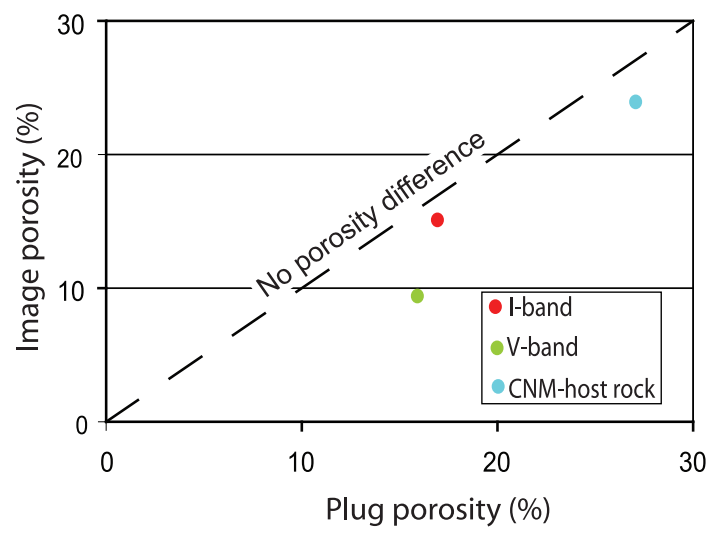

- Deformation band; approach 1 was applied.

A Deformation band; approach 2 was applied.

- Deformation band; approach 3 was applied.

Host rock; approach 2 was applied.

Figure 8. Comparison of laboratory measurements with (a) calculated permeability and (b) calculated porosity. The plugs are prepared perpendicular to the deformation bands. The calculated porosity is seen to be slightly lower, whereas the calculated permeability values from the third approach are up to two orders of magnitude lower than the laboratory measurements. The three approaches used for these measurements are addressed in Figure 6 and the results section in the text.

approaches have their limitations and uncertainties. The thin-section based image analysis technique presented here makes it possible to estimate the properties of the bands themselves. The practical importance of this technique has been demonstrated by combining the measured values of porosity and specific surface area with a Kozeny-Carman relation to obtain estimates of fluid permeability. The main cause of the errors in image processing methods is the discrepancy between thin sections and the actual rock samples; that is, a thin section is only a small part of the rock and can give slightly different statistical parameters [Keehm et al., 2004]. However, this is not the case for deformation bands because of their small thickness. Therefore, image processing techniques through thin section analysis are suitable for extracting microstructural information from deformation bands.
[46] Using image analysis techniques, the effect of magnification and resolution of the images on the calculated specific surface areas has been investigated. We have resolved the sensitivity of the image processing method to the resolution of the image by using high-resolution images that provide insight into more complex microgeometries. In order to calculate specific surface area, Blair et al. [1996] used only one high magnification image from the undeformed sample. Our study shows that a high magnification image is not appropriate for estimating the properties of deformation bands with a relatively wide range of grain and pore sizes. Because of cataclasis and other deformation processes, the microgeometry of most deformation bands is complex. Hence the use of a single high-magnification image that captures only a small area of the anisotropic medium is inappropriate. Permeability values obtained from the third approach are different from the values calculated from first and second approaches (Figure 6).

[47] The difference between the two groups of data in Figure 6 can be ascribed to the deformation mechanisms involved during the initiation and development of the bands. In the first group (samples from Sinai), only cataclasis is observed, while in the second group (samples from Utah), both cataclasis and pressure chemical dissolution are present.

[48] We have tested the concept of Berryman [1998] on stationary and anisotropic random porous media for deformed sandstones to quantify the permeability variations along the deformation band using 2D images from thin sections with different orientations. We show that the anisotropic deformation bands are not two-dimensional, i.e., the random variations occur both in the $\mathrm{xz}$ and $\mathrm{yz}$ planes (Figure 2). The main purpose of using 2D images with different orientations is to investigate porosity and permeability variations in different directions and consequently quantify the porosity and permeability anisotropy induced by deformation bands. We have identified deformation-induced anisotropy in properties such as porosity and permeability in deformed sandstones. This anisotropy occurs at both macro- and microscopic scales in the deformation bands. In this study we point out the microscopic aspect of anisotropy along deformation bands. On the basis of the presented results (Figure 7), the permeability changes along the band are substantial and the difference is almost two orders of magnitude in the two directions. The permeability values form two different clusters, with higher porosity and permeability in the yz plane. Since deformation bands are long and introduce discontinuities and compartmentalization to fluid reservoirs, studying their microstructure and variations along the bands is significant.

[49] We have used a modified version of the KozenyCarman relation to calculate permeability. In order to calculate the formation factor, the exponential relationship between formation factor and porosity obtained from an empirical relationship introduced by Archie [1942] was used. On the basis of the previous studies on the formation factor of sandstones, we have assumed the exponent $\mathrm{m}$ in equation (14) to be equal to 1.8 for our moderately consolidated sandstones in the deformation bands. Our investigation reveals that varying the exponent $\mathrm{m}$ within the range of 1.5 to 2 for deformation bands, which is the range proposed for the poorly consolidated to well consolidated sandstone [Brace, 1977; Sen et al., 1981; Wong et al., 1984; Blair et al., 
1996], does not affect the calculated permeability significantly (only a few milli-Darcy). We think the results would be more accurate if we use the lab-measured formation factor for the deformed sandstones in this equation. Our results show that both porosity and permeability decrease as a result of cataclasis and quartz dissolution in the band, but permeability changes are higher by up to three orders of magnitude. This can be explained by the increased specific surface area in the band due to the presence of the crushed grains.

[50] Considering the results obtained from application of our third approach for calculating permeability, the difference between calculated and measured permeability are substantial (up to two orders of magnitude, see Figure 8). For the porosity, the calculated porosity is to some extent less than the laboratory measurements (Figure 8). In order to quantify the overestimation of the laboratory measured porosity, we need to analyze more data. The difference between plug measurements and our calculated permeability and porosity can be explained by the sample-size limitation inherent to the plug measurements, in which it is difficult or impossible to get the properties of a millimeter-thick deformation band. The laboratory-measured data represent the average properties of the band and its host rock. The method demonstrated here represents a means of finding and mapping variations in permeability along as well as across narrow zones such as deformation bands. This opens new opportunities for research on how deformation bands and similar features affect fluid flow in three dimensions.

\section{Notation}

$S_{n} \quad n=1,2, \ldots$ N-point correlation function

$r$ spatial positions in 2D images

$f(r)$ characteristics or indicator function equal to 1 or 0 depending on the position $r$

$\phi$ porosity

$S_{2} \quad(x, y)$ two-point correlation function for distance in Cartesian coordinates

$S_{2} \quad(r, \theta)$ two-point correlation function for a distance in polar coordinates

$i, j$ pixel positions in 2D BSE images

$k$ permeability

$c$ constant related to pore geometry

$F$ formation factor

$s \quad$ specific surface area

$m$ Archie's cementation factor

$v_{g}$ grain volume

$v_{p}$ pore volume

[51] Acknowledgments. This study was completed in the frame of the Fault Facies Project at the Centre for Integrated Petroleum Research, University of Bergen, and supported by the Norwegian Research Council, Statoil, Conoco-Philips. Thanks are due to Alvar Braathen for scientific discussions. Thanks go to Egil Sev. Erichsen for help with the SEM analyses and also to Atle Rotevan, Eivind Bastesen, Tore Skar and Silje S. Berg for participating in the collection of some of the samples used in this work. We appreciate the constructive comments of the reviewers on early versions of the manuscript. We are grateful to Brian Anthony Farrelly for his help.

\section{References}

Antonellini, M., and A. Aydin (1994), Effect of faulting on fluid flow in porous sandstones: Petrophysical properties, AAPG Bull., 78, 355-377.
Archie, G. E. (1942), The electrical resistivity log as an aid in determining some reservoir characteristics, Trans. Am. Ins. Min. Metall. Pet. Eng., $146,54-62$.

Aydin, A., R. I. Borja, and P. Eichhubl (2006), Geological and mathematical framework for failure modes in granular rock, J. Struct. Geol., 28, $83-98$.

Bakke, S., and P. E. Øren (1997), 3-D pore-scale modelling of sandstones and flow simulations in the pore networks, SPE J., 2(2), 136-149.

Bense, V. F., E. H. Van den Berg, and R. T. Van Balen (2003), Deformation mechanisms and hydraulic properties of fault zones in unconsolidated sediments; The Roer Valley Rift System, The Netherlands, Hydrogeol. $J ., 11,319-332$.

Berge, P. A., J. G. Berryman, S. C. Blair, C. Pana (1997), Scalar properties of transversely isotropic tuff from images of orthogonal cross sections, UCRL-JC- Lawrence Livermore Natl. Lab., Livermore, Calif.

Berryman, J. G. (1998), Planar spatial correlations, anisotropy, and specific surface area of stationary random porous media, J. Appl. Phys., 83(3), $1685-1693$

Blair, S. C., P. A. Berge, and J. G. Berryman (1996), Using two-point correlation functions to characterize microgeometry and estimate permeabilities of sandstones and porous glass, J. Geophys. Res., 101(B9), 20,359-20,375.

Brace, W. E. (1977), Permeability from resistivity and pore shape, J. Geophys. Res., 82(23), 3343-3349.

Corson, P. (1974), Correlation functions for predicting properties of heterogeneous materials: I. Experimental measurements of spatial correlation functions in multiphase solids, J. Appl. Phys., 45, 3159-3164.

Cule, D., and S. Torquato (1999), Generating random media from limited microstructural information via stochastic optimization, J. Appl. Phys., 86(6), 3428-3437.

Darcy, H. (1856), Les Fontaines Publiques de la Ville de Dijon, 647 pp., Dalmont, Paris.

Debye, P., Jr., H. R. Anderson, and H. Brumberger (1957), Scattering by an inhomogeneous solid: II. The correlation function and its application, J. Appl. Phys., 28, 670-683.

Doyen, P. M. (1988), Permeability, conductivity, and pore geometry of sandstone, J. Geophys. Res., 93(B7), 7729-7740.

Du Bernard, X. D., P. Eichhubl, and A. Aydin (2002), Dilation bands: A new form of localized failure in granular media, Geophys. Res. Lett. 29(24), 2176, doi:10.1029/2002GL015966.

Ehrlich, R., S. K. Kennedy, S. J. Crabtree, and R. L. Cannon (1984), Petrographic image anlysis: I. Analysis of reservoir pore complexes, J. Sediment. Res., 54, 1515-1522.

Fisher, Q. J., and R. J. Knipe (2001), The permeability of faults within siliciclastic petroleum reservoirs of the North Sea and Norwegian Continental Shelf, Mar. Pet. Geol., 18, 1063-1081.

Fossen, H., R. A. Schultz, K. Mair, and Z. K. Shipton (2007), Deformation bands in sandstones-A review, J. Geol. Soc. London, 164, 754-769.

Garboczi, E. J., D. P. Bentz, and N. S. Martys (1999), Digital images and computer modelling. Experimental methods in the physical sciences, in Methods in the Physics of Porous Media, vol. 35, chap. 1, pp. 1-41, Elsevier, New York.

Gibson, R. G. (1998), Physical character and fluid-flow properties of sandstone-drived fault zones, in Structural Geology in Reservoir Characterization, edited by M. P. Coward, H. Johnson, and T. S. Daltaban, Geol. Soc. London, Spec. Publ., 127, 83-97.

Gokhale, A. M. (2004), Experimental measurements and interpretation of microstructural n-point correlation functions, Microsc. Microanal., 10(2), $736-737$.

Hesthammer, J., and H. Fossen (2001), Structural core analysis from the Gullfaks area, northern North Sea, Mar. Pet. Geol., 18, 411-439.

Jamison, W. R., and D. W. Stearns (1982), Tectonic deformation of Wingate Sandstone, Colorado National Monument, AAPG Bull., 6, 2584-2608.

Keehm, Y., T. Mukerji, and A. Nur (2004), Permeability prediction from thin sections: 3D reconstruction and Lattice-Boltzmann flow simulation, Geophys. Res. Lett., 31, L04606, doi:10.1029/2003GL018761.

Keehm, Y., K. Sternlof, and T. Mukerji (2006), Computational estimation of compaction band permeability in sandstone, Geosci. J., 10(4), 499-505.

Klinkenberg, L. J. (1941), The permeability of porous media to liquid and gases, in Drilling and Production Practice, pp. 200-213, American Petroleum Inst.

Knipe, R. J., Q. J. Fisher, G. Jones, M. B. Clennell, and A. B. Farmer (1997), Fault seal analysis: Successful methodologies, application and future directions, in Hydocarbon Seals: Importance for Exploration and Production, edited by P. Moller-Pederson and A. G. Koestler, NPF Spec. Pub., 7, 15-40.

Koplik, J., C. Lin, and M. Vermette (1984), Conductivity and permeability from microgeometry, J. Appl. Phys., 56, 3127-3131.

Kwon, O., B. T. Ngwenya, I. G. Main, S. C. Elphick (2005), Permeability evolution during deformation of siliciclastic sandstones from Moab, 
Utah, in Faults, Fluid Flow, and Petroleum Traps, edited by R. Sorkhabi and Y. Tsuji, AAPG Mem., 85, 219-236.

Lothe, A. E., R. H. Gabrielsen, N. Larsen, and B. T. Bjørnevoll (2002), An experimental study of the texture of deformation bands: Effects on porosity and permeability of sandstones, Pet. Geosci., 8, 195-207.

Ogilvie, S. R., and P. W. J. Glover (2001), The petrophysical properties of deformation bands in relation to their microstructure, Earth Planet. Sci. Lett., 193, 129-142.

Otsu, N. (1979), A threshold selection method grey-level histogram, IEEE Trans. Syst., 9(1), 62-66.

Paterson, M. S. (1983), The equivalent channel model for permeability and resistivity in fluid-saturated rock- A reappraisal, Mech. Mater., 2, 345-352.

Pittman, E. D. (1981), Effect of fault-related granulation on porosity and permeability of quartz sandstones, Simpson Group (Ordovician) Oklahoma, AAPG Bull., 65, 2381-2387.

Prodanovic, M., W. B. Lindquist, and R. S. Seright (2007), 3D image-based characterization of fluid displacement in a Berea core, $A d v$. Water Resour., 30, 214-226.

Sen, P. N., C. Scala, and M. H. Cohen (1981), A self-similar model for sedimentary rocks with application to the dielectric constant of fused glass beads, Geophysics, 46, 781-795.

Shipton, Z. K., J. P. Evans, K. R. Robeson, C. B. Forster, and S. Snelgrove (2002), Structural heterogeneity and permeability in eolian sandstone: Implications for subsurface modeling of faults, AAPG Bull., 86(5), $863-883$.
Sigda, J. M., L. B. Goodwin, P. S. Mozelyand J. L. Wilson (1999), Permeability alteration in small-displacement faults in poorly lithified sediments: Rio Grande, Central New Mexico, in Faults and Subsurface Fluid Flow in the Shallow Crust, Geophys. Monogr. Ser., vol. 113, AGU, Washington, D. C.

Sternlof, K. R., J. R. Chapin, D. D. Pollard, and L. J. Durlofsky (2004), Permeability effects of deformation band arrays in sandstone, $A A P G$ Bull., 88(9), 1315-1329.

Walsh, J. B., and W. F. Brace (1984), The effect of pressure on porosity and the transport properties of rock, J. Geophys. Res., 89(B11), 9425-9431.

White, J. A., R. I. Borja, and J. T. Fredrich (2006), Calculating the effective permeability of sandstone with multiscale lattice Boltzmann/finite element simulations, Acta Geotech., 1, 195-209.

Wissler, T. M. (1987), Sandstone pore structure: A quantitative analysis of digital SEM images, Ph.D. thesis, Mass. Inst. of Technol., Cambridge.

Wong, P. Z., J. Koplik, and J. P. Tomanic (1984), Conductivity and permeability of rocks, Phys. Rev., B30, 6606-6614.

Wyllie, M. R. J. (1953), Formation factors of unconsolidated porous media: Influence of particle shape and effect of cementation, Trans. AIME, 198, $103-110$

B. Alaei, Rocksource ASA, Olav Kyrresgt. 22, 5808 Bergen, Norway. (Behzad.Alaeimajolan@rocksource.com)

H. Fossen and A. Torabi, Department of Earth Science and Centre for Integrated Petroleum Research, University of Bergen, Allégt. 41, 5007 Bergen, Norway. (haakon.fossen@geo.uib.no; anita.torabi@cipr.uib.no) 